ARTICLE

\title{
Crystallographic structure of wild-type SARS-CoV- 2 main protease acyl-enzyme intermediate with physiological C-terminal autoprocessing site
}

Jaeyong Lee ${ }^{1,2,4}$, Liam J. Worrall ${ }^{1,4}$, Marija Vuckovic ${ }^{1}$, Federico I. Rosell1, Francesco Gentile ${ }^{3}$, Anh-Tien Ton (1) ${ }^{3}$, Nathanael A. Caveney (1D ${ }^{1}$, Fuqiang Ban ${ }^{3}$, Artem Cherkasov (1) ${ }^{3}$, Mark Paetzel (D) ${ }^{2 凶} \&$

Natalie C. J. Strynadka (i) ${ }^{1 凶}$

Severe Acute Respiratory Syndrome Coronavirus 2 (SARS-CoV-2), the pathogen that causes the disease COVID-19, produces replicase polyproteins 1a and 1ab that contain, respectively, 11 or 16 nonstructural proteins (nsp). Nsp5 is the main protease (Mpro) responsible for cleavage at eleven positions along these polyproteins, including at its own $\mathrm{N}$ - and $\mathrm{C}$-terminal boundaries, representing essential processing events for subsequent viral assembly and maturation. We have determined X-ray crystallographic structures of this cysteine protease in its wild-type free active site state at $1.8 \AA$ resolution, in its acyl-enzyme intermediate state with the native $\mathrm{C}$-terminal autocleavage sequence at $1.95 \AA$ resolution and in its product bound state at $2.0 \AA$ resolution by employing an active site mutation $(C 145 A)$. We characterize the stereochemical features of the acyl-enzyme intermediate including critical hydrogen bonding distances underlying catalysis in the Cys/His dyad and oxyanion hole. We also identify a highly ordered water molecule in a position compatible for a role as the deacylating nucleophile in the catalytic mechanism and characterize the binding groove conformational changes and dimerization interface that occur upon formation of the acylenzyme. Collectively, these crystallographic snapshots provide valuable mechanistic and structural insights for future antiviral therapeutic development including revised molecular docking strategies based on Mpro inhibition.

\footnotetext{
${ }^{1}$ Department of Biochemistry and Molecular Biology and Centre for Blood Research, The University of British Columbia, Vancouver, BC, Canada.

2 Department of Molecular Biology and Biochemistry, Simon Fraser University, Burnaby, BC, Canada. ${ }^{3}$ Vancouver Prostate Centre, The University of British

Columbia, Vancouver, BC, Canada. ${ }^{4}$ These authors contributed equally: Jaeyong Lee, Liam J. Worrall. ${ }^{\varpi_{e}}{ }$ mail: mpaetzel@sfu.ca; ncjs@mail.ubc.ca
} 
S ARS-CoV-2 main protease ( $\mathrm{M}^{\mathrm{pro}}$ ) is one of two cysteine proteases necessary for viral replication and assembly, with analogous functional counterparts in earlier SARS-CoV-1 and Middle Eastern Respiratory Syndrome (MERS) coronavirus variants ${ }^{1}$. SARS-CoV-2 $\mathrm{M}^{\text {pro }}$ is 306 residues in length corresponding to residues 3264-3569 within the large polyproteins ppla or pplab (generated by a ribosomal frameshift during translation). It is denoted by enzyme commission number EC: 3.4.22.69 within the Merops Database Classification subclan PA (C) and family C30. Initial structures of SARS-CoV-1 Mpro ( $\sim 96 \%$ identical to SARS-CoV-2 Mpro) showed a dimer with each protomer composed of a double-barreled catalytic region, with structural similarity to $3 \mathrm{C}$ protease found in picornaviruses (and thus its alternate name $3 \mathrm{C}$-like protease $3 \mathrm{CL}^{\text {pro }}$ ), that is followed by an all $\alpha$-helical domain ${ }^{2,3}$. The structures also suggested the enzyme employs a cysteine (Cys145) side chain thiolate as a nucleophile in the presumed initial acylation step of peptide bond cleavage, potentially assisted by an adjacent histidine (His41) in the enzyme active site (see Supplementary Fig. 1a for reaction schematic). Mpro cleavage of ppla and pplab at the 11 sites, including autoprocessing sites at its own $\mathrm{N}$ - and C-termini, releases nonstructural proteins (nsp) 4-16. SARS-CoV-1 Mpro has been shown to proteolyze dodecapeptides spanning each of the 11 processing sites (Supplementary Fig. 1b), cleaving after glutamine in the consensus sequence (P2:Leu/Met/Phe/Val)-P1: $\mathbf{G l n} \downarrow-(\mathbf{P 1}$ ':Ser/Ala/Gly/Asn) with the two peptides corresponding to the $\mathrm{N}$ - and $\mathrm{C}$-terminal $\mathrm{M}^{\mathrm{p}}$ ro autocleavage sites having the highest efficiency ${ }^{4}$.

Although crystallographic structures of SARS-CoV-2 Mpro are compounding weekly in the literature, in native forms $\mathrm{s}^{5-7}$ and with various bound chemical fragments ${ }^{5}$ or inhibitors ${ }^{7-10}$, a missing link for SARS-CoV-2 Mpro and indeed its SARS-CoV-1 and MERS-CoV relatives, remains the lack of atomic resolution information for key intermediary mechanistic steps with native active site and physiological substrate(s). To that end, in this paper, we present the structure, at $1.95 \AA$ Aresolution, of the wildtype acyl-enzyme intermediate of SARS-CoV-2 Mpro covalently bound to its natural autocatalytic processing site at its Cterminus. Capture of this intermediate provides atomic details of the acyl-enzyme coordination geometry and stabilization, the surrounding solvation/desolvation, as well as the underlying substrate specificity determined by side chain type and orientation of the P1-P6 residues-SGVTFQ - with those of the complimentary pockets in the Mpro active site. Further, a product complex of the same substrate, captured at $2.0 \AA$ resolution using a Cys145Ala mutation, is also presented, providing further mechanistic and atomic information to inform future therapeutic design.

\section{Results}

Purification of an active Mpro dimer. Recombinant SARS-CoV$2 \mathrm{M}^{\text {pro }}$ with native $\mathrm{N}$ - and $\mathrm{C}$-termini ${ }^{11}$ was overexpressed and purified with slight modifications of previous protocols ${ }^{9}$. We also produced the catalytic mutant $\mathrm{C} 145 \mathrm{~A}$ and a mutant that impacts dimerization, P9T, with similar protocols, see "Methods". Biological small-angle X-ray scattering (bioSAXS) and SEC-MALS demonstrate the wild-type protein is exclusively a dimer across a range of protein and salt concentrations, while the P9T mutant is predominantly monomeric (Supplementary Figs. 2 and 3). Based on the published analytical ultracentrifugation results, SARSCoV-2 Mpro is known to exhibit a high propensity for dimerization with a $K_{\mathrm{D}} \sim 2.5 \mu \mathrm{M}$ (ref. ${ }^{7}$ ). Dimerization, with the two protomers associated at right angles to form a heart-shaped complex (Fig. 1a, b), has been shown to be critical for efficient catalytic activity in SARS-CoV-1 (ref. ${ }^{12}$ ), with the interface
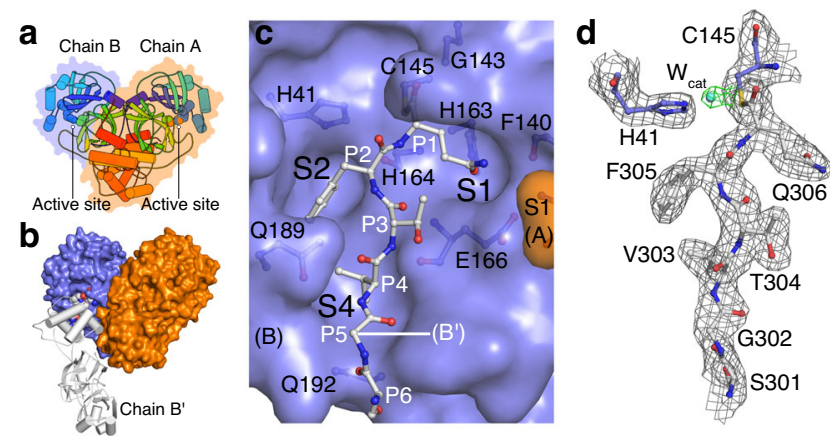

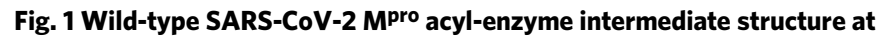
1.95 Å resolution. a Overview of $M^{\text {pro }}$ dimer. Each protomer colored spectrally ( $\mathrm{N}$-terminus blue to $\mathrm{C}$-terminus red). A transparent molecular surface is shown around each protomer (chain A-orange, chain B-blue). b $M^{\text {pro }}$ structure determined here shown in molecular surface colored as in (a). A symmetry-related chain in the crystal lattice $\left(B^{\prime}\right.$, white) directs its C-terminal six residues into the substrate binding groove of chain $B$ (Ser301-GIn306 shown in CPK space filling representation). c Substrate binding groove (blue surface) of chain $B$ with covalently bound $C$-terminal P1-P6 residues of $\mathrm{B}^{\prime}$. The $\mathrm{N}$-terminus of chain $\mathrm{A}$ (Ser1, the so-called $\mathrm{N}$ finger, orange surface) provides structural support for the $\mathrm{S} 1$ pocket of chain B. The side chains of the catalytic residues Cys145, His41, and residues that make direct hydrogen bonds to substrate are shown. $\mathbf{d} 2 \mathrm{mF}_{\mathrm{o}}$ $\mathrm{DF}_{\mathrm{c}}$ electron density contoured at $1.0 \sigma$ around the chain $\mathrm{B}^{\prime} \mathrm{C}$-terminus clearly reveals the thioester bond. Electron density for $W_{\text {cat }}$ adjacent to the thioester carbonyl carbon shown in green, also contoured at $1.0 \sigma$. A simulated annealing OMIT map for the bound substrate is shown in Supplementary Fig. 5a.

interactions required for correct active site structure, including notably the $\mathrm{N}$-terminus (N-finger, Ser1) of each protomer stabilizing the S1 substrate binding pocket of its neighboring protomer $^{2,3}$ (Fig. 1c, orange surface). The wild-type Mpro preparation used for our structural analysis is active in a FRET-based assay with enzymatic parameters and inhibition by the antineoplastic agent carmofur $\left(\mathrm{IC}_{50}\right.$ value of $\left.1.8 \pm 0.3 \mu \mathrm{M}\right)$ consistent with those previously determined ${ }^{8}$ (Supplementary Fig. 4). As also observed elsewhere ${ }^{13}$, the determined Hill coefficient was greater than one, indicating positive cooperativity. Possible explanations for this, which future studies may unravel, include allosteric communication between the two active sites within the dimer upon substrate binding or, alternatively, a substrateinduced dimerization. Mutation of the catalytic cysteine to alanine $(\mathrm{C} 145 \mathrm{~A})$ abolishes activity, while the dimerization defective P9T (with native active site) lowers the catalytic efficiency by $>50$ fold (Supplementary Fig. 4c).

Crystallographic determination of Mpro in complex with its Cterminal autocleavage sequence. Using $\mathrm{X}$-ray crystallography, we have captured two unique structures of SARS-CoV-2 $\mathrm{M}^{\mathrm{p}}$ io in complex with its C-terminal autocleavage site in trans, representative of distinct enzyme mechanistic states. First, an acylenzyme intermediate with the C-terminal residues bound in the active site of a neighboring dimer and Gln306 covalently bound to catalytic Cys145 in the wild-type protein and, second, a product-like form with the same C-terminal autocleavage sequence observed bound non-covalently in the active site of a catalytically inactive C145A mutant.

Wild-type and C145A Mpro were crystallized at $\mathrm{pH} 6$ in space group C2 with isomorphous unit cell dimensions (Supplementary Table 1). For both structures, the asymmetric unit is composed of an Mpro dimer with crystal packing orienting the C-terminus of one monomer (chain $\mathrm{B}^{\prime}$ ) proximal to the active site of a 

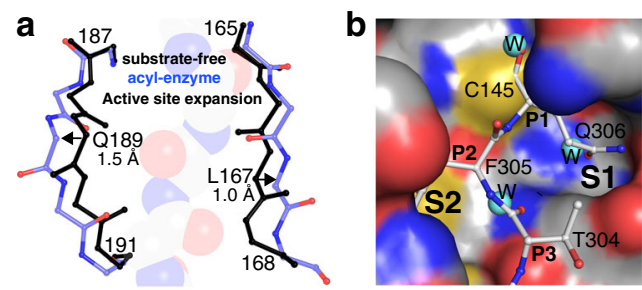

C

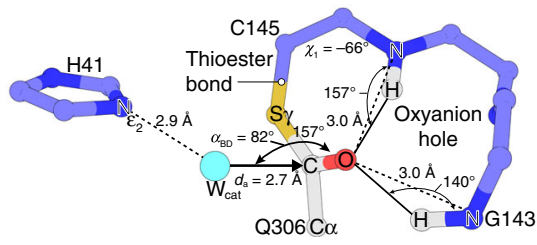

Fig. 2 Comparison of wild-type acyl-enzyme intermediate and substratefree $\mathbf{M}^{\text {pro }}$ structures. a Superposition of the substrate-free (black) and acyl-enzyme (blue) forms reveals changes in the substrate binding groove width. The main chain atoms for bound $\mathrm{B}^{\prime}$ substrate are shown as transparent van der Waals spheres. b Molecular surface of wild-type Mpro with three-ordered water molecules (cyan spheres). Superposition of the acyl-enzyme structure shows these waters are coincident with oxygen atom positions and will be displaced upon substrate binding. c Analysis of the wild-type acyl-enzyme active site reveals a potential deacylating water (catalytic/nucleophilic- $W_{\text {cat }}$ ) approaching the Re-face of the thioester. Ball and stick diagram depicting the geometry and atomic interactions of the thioester linkage between the Sy of Cys 145 and main chain carbonyl carbon of substrate Gln306. The trigonal planar nature of the thioester group, defined by atoms $\mathrm{C} \alpha, \mathrm{C}$, and $\mathrm{O}$ of $\mathrm{Gln} 306$, and $\mathrm{S} \gamma$ of Cys145 is shown as is the $\chi_{1}$ dihedral angle (defined by atoms $N, C \alpha, C \beta$, and $S \gamma$ ). The oxyanion hole hydrogen bond distances and angles are also labeled. Proposed deacylating water $\left(W_{\text {cat }}\right)$ shown as a cyan sphere. $\alpha_{\mathrm{BD}}$ is the Bürgi-Dunitz angle $\left(W_{\text {cat }}{ }^{-} C=O\right)$ and $d_{\mathrm{a}}$ the attack distance.

symmetry-related monomer (chain B; Fig. 1b). In the mature enzyme (residues 1-306), the C-terminal autocleavage sequence Ser301-Gln306 packs at the dimerization interface as observed in chain A; however, in chain B it is instead rotated almost $180^{\circ}$ toward domain III and inserted into the neighboring active site, occupying the S6-S1 substrate binding pockets (Fig. 1c). This results in one protomer with substrate bound and one empty in each dimer pair. For the wild-type acyl-enzyme intermediate complex, there is clear continuous density showing the carbonyl carbon atom of the C-terminal Gln306 covalently bound to the sulfur atom of catalytic Cys145 (Fig. 1d and Supplementary Fig. 5a). The C-terminal autocleavage site binds within the substrate binding groove in an extended conformation (Figs. 1c and $2 \mathrm{a}, \mathrm{b})$, making antiparallel $\beta$-sheet, as well as side chainmediated hydrogen bond interactions with residues 164-166 of $\beta$ strand 12 (see Supplementary Fig. 6 for numbering) on one side, and with residues $189-191$ of the $\sim 15$ residue loop linking domains II and III on the other (Fig. 2a and Supplementary Fig. 7a). For the C145A mutant product-like complex, the Cterminus binds in the same extended manner, forming analogous main chain and side chain interactions (Supplementary Fig. 7b). Well-ordered electron density unambiguously confirms the alanine mutation and presence of terminating main chain carboxylate oxygens (Fig. 3a and Supplementary Fig. 5b).

Characterization of the Mpro acyl-enzyme intermediate complex with physiological substrate. The $1.95 \AA$ resolution structure of the SARS-CoV-2 Mpro provides atomic details of the fully resolved acyl-enzyme intermediate state central to the catalysis of ppla/ab processing during viral maturation and pathogenicity. The electron density clearly defines the stereochemistry of the a

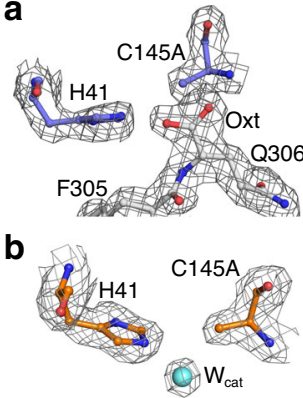

C

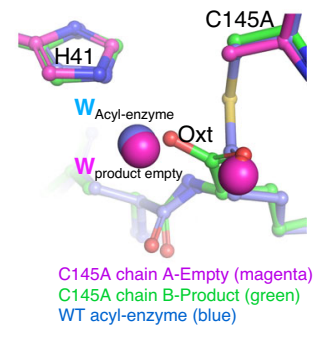

Fig. 3 C145A SARS-CoV-2 Mpro product complex at $2.0 \AA$ resolution. a $2 \mathrm{mF}_{\mathrm{o}}-\mathrm{DF}_{\mathrm{c}}$ electron density (contoured at $1.0 \sigma$ ) in chain $\mathrm{B}$ of the $\mathrm{C} 145 \mathrm{~A}$ mutant shows presence of the bound $\mathrm{C}$-terminal product of symmetryrelated molecule $B^{\prime}$. Also see Supplementary Fig. 5b. b $2 \mathrm{mF}_{\mathrm{o}}-\mathrm{DF}_{\mathrm{c}}$ electron density $(1.0 \sigma)$ of empty protomer, chain $A$, of the same C145A mutant structure shows presence of a highly ordered water molecule hydrogen bonded to $\mathrm{N} \varepsilon 2$ of His41, consistent with a general base role of the latter and coincident in position with the $W_{\text {cat }}$ weakly observed in the acyl-enzyme complex as in c and Fig. 1c. c Superposition of the product chain A (empty binding site; magenta) and chain B (product bound; green) with the acylenzyme (chain B; blue).

thioester bond formed between the Cys145 side chain $\gamma$-sulfur atom and the carbonyl carbon of its (self) substrate at the residue preceding the scissile bond position $\mathrm{Gln} 306$ (P1) as trigonal planar $(\mathrm{S} \gamma-\mathrm{C}-\mathrm{O}-\mathrm{C} \alpha)$ and with a Cys145 $\chi_{1}$ angle of $-66^{\circ}$. The carbonyl oxygen of the intermediate is stabilized by bifurcated hydrogen bonds with the main chain nitrogens of Cys145 and Gly143 at distances for both of $3.0 \AA$ (Fig. 2c and Supplementary Fig. 7a), providing an ideal oxyanion hole interaction. The closest approach of the thioester sulfur of Cys145 to the potential general acid/base His41 is a distance of $3.7 \AA$ to its Ne2, and at an angle not in keeping with a direct hydrogen bond. The general disposition of the Cys145 S $\gamma$ to substrate P1 carbonyl delineate a Reface attack in the acylation step (Fig. 2c). The His41 imidazole is packed between the side chains of Pro39 and Met49, with N $\delta 1$ hydrogen bonding to a highly ordered, multi-coordinated (His164, Asp187 side chains), and buried water molecule, previously proposed to play a role in regulating the protonation state of His41 in SARS-CoV-1 via QM/MM calculations ${ }^{14}$. The solvent accessible surface of the His41 imidazole Ne2 calculated by Areaimol $^{15}$ is almost fully buried, with a value of $1.4 \AA^{2}$, as compared to the free active site, $7.9 \AA^{2}$, a factor likely influencing the $\mathrm{p} K_{\mathrm{a}}$, protonation state, and potential role in catalysis.

Anchored by the covalent thioester bond to Cys145, oxyanion hole coordination, and extended $\beta$-sheet hydrogen bonding of substrate and active site, the P1-P6 (QFTVGS) specificity determinants are accommodated within the inward facing enzyme cleft pockets S1, S2, and S4 (Fig. 1c), providing multiple stabilizing noncovalent interactions (Supplementary Fig. 7a) and collectively $512 \AA^{2}$ of buried enzyme surface. Notably, the presence of a phenylalanine in the $\mathrm{P} 2$ position results in a wider binding pocket compared to the empty active site forms, created by shifts of the side chains of Met165 and Gln189 to accommodate, with the side chain amide of the latter redirecting to form stabilizing hydrogen bonds with the $\mathrm{P} 4$ main chain atoms (Fig. 1c and Supplementary Fig. 7). Overlap of the acyl-enzyme intermediate structure with the wild-type substrate-free structure (that determined here, as well as the highest resolution published structure PDB 6YB7) shows that the binding of substrate results in a significant outward shift and increase of overall width of the substrate binding groove (Fig. 2a). Residues making up the outer edge of the binding site adjust up to $1.5 \AA$ on one side (residues 187-191) and up to $1.0 \AA$ on the other side (residues 165-168), 
both regions which directly bind substrate, suggesting an unusual expansion of the binding groove rather than constriction typical of most serine proteases ${ }^{16}$ is required for the C-terminal autoprocessing substrate to optimally fit into the Mpro active site. Interestingly, if the same overlap is done with the empty protomer (chain A) in the acyl-enzyme intermediate or product complex dimers, this expansion is only observed for residues 187-191 and to a lesser degree, suggesting possible allosteric communication between the two protomers of the active dimeric form upon substrate binding.

Important aspects of solvation/desolvation in formation of the acyl-enzyme intermediate are also interpreted from our data. Displacement of three highly ordered water molecules visible in the wild-type substrate-free structure occupy the position of the substrate carboxyl oxygen in the oxyanion hole, the Gln306 (P1) side chain oxygen, and Thr304 (P3) main chain carbonyl oxygen (Fig. 2b). These same highly ordered water molecules are typically observed in other SARS-CoV-2 Mpro structures, including the highest resolution structure yet reported at $1.25 \AA$ (ref. ${ }^{5}$; PDB 6YB7), although in that case a DMSO molecule is observed in the P1 site with the sulfoxide oxygen overlapping the water binding location. Notably, an additional, unique water position, in keeping with that of a deacylating water nucleophile, is observed in the acyl-enzyme intermediate structure, with weak but significant density (Fig. 1d). Positioned orthogonally with respect to the other atoms in the trigonal planar thioester group (Fig. 2c), the $1.95 \AA$ resolution allows for a measurement of the approximate attack angle that the oxygen of this water (upon activation, the hydroxide anion, $\mathrm{OH}^{-}$) would take relative to the substrate carbonyl carbon. The angle, often termed the Bürgi-Dunitz angle $\left(\alpha_{\mathrm{BD}}\right)^{17}$, is defined by three atoms: the nucleophile (in this case a water oxygen $\mathrm{O}$ ), the carbonyl carbon $\mathrm{C}$, and the carbonyl oxygen $\mathrm{O}(\mathrm{O}---\mathrm{C}=\mathrm{O}$ angle), with the generalized ideal falling near $107^{\circ}$. The putative deacylating water sits approximately equidistant between the $\mathrm{N \varepsilon} 2$ of His41 in the acyl-enzyme (2.9 $\AA$ ), reinforcing its role as the activating general base, and the scissile carbonyl carbon (2.7 $\AA$ ) and with a Bürgi-Dunitz angle as above of $\sim 82^{\circ}$. Displacement of catalytic water molecules is a reoccurring theme in structure-based drug discovery and the observation of this water position in the context of the acyl-enzyme intermediate is an important advance in this regard (discussed further below).

Given the typically short-lived nature of the acyl-enzyme catalytic intermediate during proteolysis, capturing these has been historically challenging. There has been some previous success in characterizing acyl-enzyme species at the atomic level in serine proteases, but these experiments typically required some level of impairment to the enzyme ${ }^{18-20}$, substrate (non-hydrolyzable adduct, poor substrates, or inhibitors), and/or conditions ${ }^{21-24}$ for a stable acylation to be observed in the crystal structure. No prior examples in the classic cysteine protease families proper have been published, although a putative cysteine protease-like glutathione hydrolase acylated with glutathione substrate captured at $\mathrm{pH} 3$ has been described ${ }^{25}$ (histidine base deprotonation highly disfavored at this extreme $\mathrm{pH}$ ). Here, the trans acylenzyme complex of full-length, wild-type Mpro is observed in the crystallographic lattice with the endogenous P1-P6 C-terminal product of symmetry-related molecule $\mathrm{B}^{\prime}$ binding into the active site cleft of molecule $\mathrm{B}$. The reaction the enzyme has catalyzed within the crystal is the reverse reaction, it is presented with the product (the free carboxylate of $\mathrm{Gln} 306$ (P1) from $\mathrm{B}^{\prime}$ ), and it has formed the acyl-enzyme by creating the thioester with the nucleophile Cys145 (see Supplementary Fig. 1a for reaction schematic). It is possible that the local effective concentration of the product as afforded by the crystal lattice has contributed to driving the reaction backward to the form the thioester. The crystals were grown at $\mathrm{pH} 6$, theoretically not low enough to prevent a solvent accessible histidine $\left(\mathrm{p} K_{\mathrm{a}} \sim 6.5\right)$ from functioning as a general base to activate a deacylating/nucleophilic water, but certainly disabling optimal activity (estimated kinetically at $<50 \%$ in SARS-CoV-1 using a pentadecapeptide substrate spanning the C-terminal cleavage site ${ }^{26}$ ). Given the observed putative deacylating water, with appropriate distances of histidine base to water to thioester intermediate, we can only further speculate that the slightly less than optimal angle of attack by the nucleophilic water, $\sim 82^{\circ}$ instead of the theoretical optimum of $107^{\circ}$, as predicted by Bürgi-Dunitz could also contribute to the intermediate capture here.

Crystallographic structure of the SARS-CoV-2 Mpro C145A product complex with physiological substrate. Capture of a well-ordered product complex in the catalytically impaired SARSCoV-2 Mpro C145A mutant is clearly defined in the electron density maps (Fig. 3a and Supplementary Fig. 5b). One oxygen of the terminating carboxylate sits coincident with that of the carbonyl of the thioester acyl-enzyme intermediate structure, forming hydrogen bonded interactions with the oxyanion hole main chain nitrogens of 2.9 and $3.0 \AA$ (Fig. $3 c$ and Supplementary Fig. $7 \mathrm{~b}$ ). The second carboxylate oxygen is positioned to form a strong inline hydrogen bond interaction with His41 Nع2 (2.9 $)$, again supporting a role of the latter in general base activation of a nucleophilic water to form such a product. In that context, the active site of the empty protomer in the C145A structure reveals electron density for five water molecules, including one not observed in the wild-type substrate-free protomer active site and lying completely coincident with the proposed deacylating water position in the acyl-enzyme intermediate above, only observed at even greater occupancy (Fig. 3b, c). The ordered water ( $B$-factor $=30 \AA^{2}$ ), is again positioned orthogonally with respect to the other atoms in the trigonal planar thioester group and with a near identical Bürgi-Dunitz angle as verified by superposition of the substrate-free active site of the $\mathrm{C} 145 \mathrm{~A}$ and native acyl-enzyme structures (Fig. 3c). We note a structure of SARS-CoV-1 Mpro $\mathrm{C} 145 \mathrm{~A}$ in a product complex with its C-terminal autocleavage site at $2.8 \AA$ resolution has been published previously ${ }^{27}$; however, potentially due to the lower resolution a catalytic water was not observed in that case.

Model of the SARS-CoV-2 Mpro enzyme-substrate complex. An interesting aspect of viral polyprotein processing proteases, including SARS-CoV-1 and $-2 \mathrm{M}^{\text {pro, }}$, are the requisite selfcleavage events to excise itself from precursor polyproteins result in a retained C-terminal product (P1-P6 as captured here) that could potentially act as a competitive inhibitor. By contrast, the $\mathrm{N}$-terminal autoprocessing P1-P6 sequence (and C-terminal end of nsp4) departs after cleavage. Considering the Mpro consensus cleavage sequence (P2:Leu/Met/Phe/Val)-P1:Gln $\downarrow-(\mathbf{P 1}$ ':Ser/Ala/ Gly), 9 out of the 11 in SARS-CoV-2 have a leucine in the P2 position, including the $M^{\text {pro }} \mathrm{N}$-terminal (nsp4-nsp5) autoprocessing sequence (Supplementary Fig. 1b). Structures of SARSCoV-1 Mpro in complex with the N-terminal sequence ${ }^{28}$ or of SARS-CoV-2 Mpro in complex with peptidomimetic inhibitors based thereon ${ }^{8}$ reveal the S2 subsite undergoes dramatic changes when it binds leucine in the $\mathrm{P} 2$ position, predominantly mediated by rearrangement of Met49 and Gln189, and surrounding regions. By contrast, the $\mathrm{M}$ pro $\mathrm{C}$-terminal autocleavage site in both SARS-CoV-1 and -2 is the only instance where there is a P2 phenylalanine which, when bound to the S2 subsite as observed here, maintains a more open conformation similar to the empty active site, albeit with movement of Met165 creating a deeper pocket. A structure of a SARS-CoV-1 Mpro C145A mutant in 

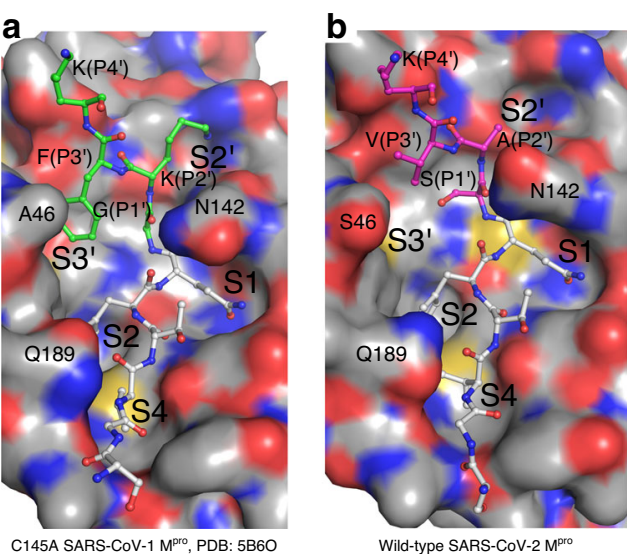

Fig. 4 Modeling of the SARS-CoV-2 $M^{\text {pro }}$ enzyme-substrate complex. a CPK molecular surface of SARS-CoV-1 C145A catalytic mutant ES complex (PDB 5B60), including C-terminal cleavage site P6-P4' (P1'-P3' with green carbons). $\mathbf{b}$ CPK molecular surface for the SARS-CoV-2 Mpro acyl-enzyme active site. The additional residues $\mathrm{P}^{\prime}-\mathrm{P} 4^{\prime}$ (magenta carbons) are modeled based on (a). Sequence alignment for all $M^{\text {pro }}$ processing sites shown in Supplementary Fig. 1b. Note the identical sequence preceding the scissile bond between SARS-CoV-1 and -2 Mpro, but divergence in $\mathrm{P}^{\prime}-\mathrm{P3}^{\prime}(\mathrm{N}-$ terminus of the subsequent nsp6). Despite these differences, the $\mathrm{S1}^{\prime}-\mathrm{S} 3^{\prime}$ pockets observed in the SARS-CoV-2 Mpro acyl-enzyme active site are similar to that in (a), i.e., already preformed in the absence of $\mathrm{P}^{\prime}-\mathrm{P}^{\prime}$ (modeled here), and apparently not dependent on the binding of $\mathrm{P} 2^{\prime}$. It is also evident from this panel that the $P 1^{\prime}-\mathrm{P}^{\prime}$ side chains are not sterically matched to the $\mathrm{S1}^{\prime}-\mathrm{S}^{\prime}$ pockets, perhaps an advantage in protein maturation.

complex with its C-terminal prosequence at $2.2 \AA$ resolution has been published previously ${ }^{29}$, representing an enzyme-substrate (ES) Michaelis-like complex (Fig. 4a). With Phe305 (P2) bound in the S2 subsite, Phe309 ( $\left.\mathrm{P3}^{\prime}\right)$ was observed to bind in an adjacent complimentary pocket with mutation of Phe309 (P3') reducing $\mathrm{C}$-terminal cleavage. This multivalent binding interaction in S2 and the $\mathrm{S}^{\prime}$ subsites was proposed to be needed to allow highaffinity binding of the $\mathrm{C}$-terminal prosequence, supported by the $10 \times$ lower-affinity binding of the C-terminal P1-P4 sequence with Phe (P2) than the equivalent $\mathrm{N}$-terminal sequence with Leu $(\mathrm{P} 2)^{29}$. Although it was further suggested that Phe309 S3' binding would be needed to order the adjacent S2 subsite, thus potentially avoiding autoinhibition by the retained post-cleavage mature $\mathrm{C}$ terminal sequence, the structures here show that these subsites are equivalently in place in the substrate-free native, acyl-enzyme intermediate and product complexes (Fig. 4).

Based on the wild-type acyl-enzyme intermediate structure here, we have generated a model of the SARS-CoV-2 ES Cterminal complex, extending from P6-P1 the downstream residues encompassing $\mathrm{P}^{\prime}-\mathrm{P} 4^{\prime}$ (Fig. $4 \mathrm{~b}$ ). Although the $\mathrm{P}^{\prime}-\mathrm{P}^{\prime}$ positions in the SARS-CoV-2 C-terminal processing site are distinct compared to SARS-CoV-1 (SAV vs GKF, respectively), the structure of the $\mathrm{S}^{\prime}$ subsite region is near identical (RMSD = $0.363 \AA$ on 50 common atoms) with the only differences compared to SARS-CoV-1 an A46S substitution on one edge of the cavity that could provide, along with the Ser307 $\left(\mathrm{P1}^{\prime}\right)$, van der Waals interactions with the smaller $\mathrm{P}^{\prime}$ valine (Fig. $4 \mathrm{~b}$ ). From the model, it is apparent that Val309 (P3') has less optimal complementarity for the broad $\mathrm{S}^{\prime}$ pocket compared to the bulky aromatic Phe309 (P3') of SARS-CoV-1. The smaller hydrophobic side chain may be needed to accommodate binding to $\mathrm{S}^{\prime}$ in the presence of the spatially adjacent $\mathrm{P}^{\prime}$ Ser. In SARS-CoV-1, $\mathrm{P} 1^{\prime}$ is a uniquely observed glycine (Supplementary Fig. 1b), providing the main chain torsion and lack of a side chain needed to be sterically compatible with the bulkier phenylalanine occupying the $\mathrm{S} 3^{\prime}$ subsite $^{28}$. Regardless, the potential buried surface of adjacent SARS-CoV-2 $\mathrm{P}^{\prime}$ Ser and $\mathrm{P}^{\prime}$ Val provides noncovalent interactions, presumably sufficient to facilitate the multivalent prime side subsite binding along with Phe305 (P2) to promote cleavage ${ }^{29}$. Further, His41 Ne2 is readily positioned inline and within hydrogen bond distance of the $\mathrm{P}^{\prime}$ main chain nitrogen, supporting its general base role in leaving group protonation during acylation and in keeping with recent QM/MM studies ${ }^{30}$. In addition, we note the $\mathrm{P} 1^{\prime}$ substitution of a serine as described above places its side chain hydroxyl adjacent and within hydrogen bonding distance to His41 Ne2.

The side chain amide of Asn142 also appears to be a point of conformational plasticity: in the acyl-enzyme intermediate and product complexes, with nothing bound in $\mathrm{S}^{\prime}$, it is swung towards that subsite, however, in the ES complex with occupied $\mathrm{S2}^{\prime}$, this rotamer would be sterically prohibited and instead is redirected to stack over the P1 Gln306 in the SARS-CoV-1 ES complex structure (Fig. 4a), potentially reinforcing binding of this key subsite and ensuring maximal substrate affinity only when $\mathrm{S2}^{\prime}$ is occupied, and in turn disfavoring autoinhibition by C-terminal product. Finally, we also observe some small conformational differences in substrate binding between the SARS-CoV-1 ES complex and our structures here. These include the main chain atoms and rotameric state of the Val303 (P4) side chain and adjacent Met165 side chain upon which it packs, potentially perturbed by the amide side chain rotamer and main chain contacts of Gln189 in the SARS-1-CoV ES complex structure.

Implications for drug discovery. SARS-CoV-2 Mpro is a major focus of antiviral drug discovery to treat COVID-19. The structures reported here provide critical information on targeting the mechanistic features and active site structure, as well as a variably exposed pocket at the dimerization interface described below.

The Mpro active site is necessarily malleable to accommodate binding of the 11 endogenous cleavage targets. In particular, the S2 subsite is significantly altered when bound to the N-terminal autoprocessing sequence with Leu (P2), as observed in complex with a SARS-CoV-1 Mpro H41A mutant ${ }^{28}$ (referred to as Leu-S2 like) compared to that captured here in complex with the wildtype SARS-CoV-2 C-terminal autoprocessing sequence (referred to as Phe-S2 like). Echoing the substrate diversity, varied hydrophobic substituents in previously characterized inhibitors have been found to bind the $\mathrm{S} 2$ site $^{7,8,10,31}$. For example, designed peptidomimetic covalent aldehyde inhibitors $11 \mathrm{a}$ and $11 \mathrm{~b}$ differ only in their P2 substituent with cyclohexyl or 3-fluorophenyl moieties, respectively (Fig. 5b, c and Supplementary Fig. 8). The cyclohexyl group resembles the binding of leucine, stacking with the His41 side chain, and induces a Leu-S2-like orientation of Met49. Conversely, the 3-fluorophenyl of $11 \mathrm{~b}$ superposes near perfectly with Phe305 (P2) in our structure with a S2 subsite correspondingly in a Phe-S2 conformation. Both are promising lead inhibitors with $\mathrm{IC}_{50}$ values $\sim 0.05 \mu \mathrm{M}$ supporting drug design strategies targeting both S2 site conformations. Given the shared Phe (S2) and Phe $\left(\mathrm{S}^{\prime}\right)$ binding sites in the SARS-CoV-1 ES complex (Fig. 4a), and observation here that this site is preformed even in the absence of prime side residues, exploring the $\mathrm{S}^{\prime}$ pocket represents a promising approach to improve inhibitors binding the Phe-S2 site. The most active noncovalent inhibitor of SARS-CoV-1 or -2 Mpro reported to date, compound $17 \mathrm{a}$, is a derivative of a compound observed to bind both the Phe-S2 and S3' sites of SARS-CoV-1 Mpro (refs. ${ }^{32,33}$ ). In absence of an experimental structure, we docked 17a to the active site of the SARS-CoV-1 ES-like complex (PDB 5B6O). Superposing also with the SARS-CoV-2 ES complex model shows that one of the 


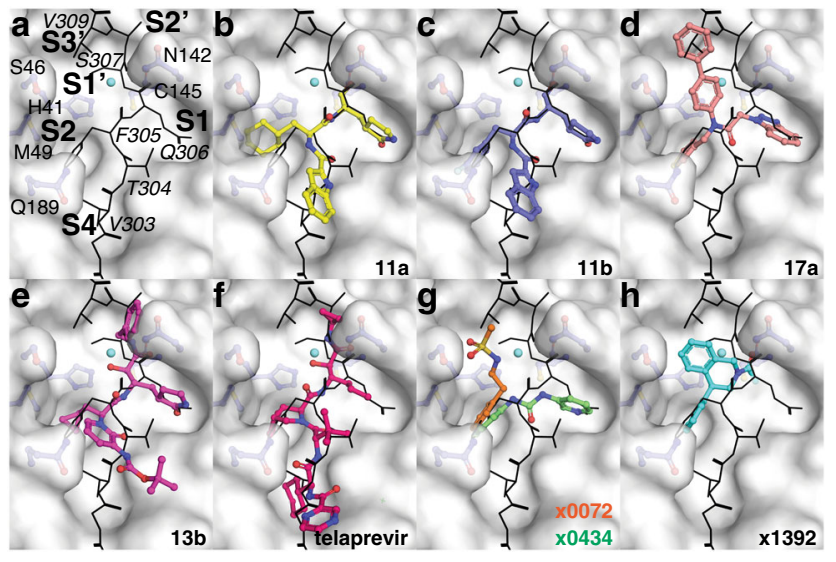

Fig. $5 \mathbf{M}^{\text {pro }}$ inhibitor binding in relation to the enzyme-substrate complex model. The surface in each panel is that of chain B of the acyl-enzyme structure. The $\mathrm{C}$-terminal autocleavage site enzyme-substrate complex model for SARS-CoV-2 (see Fig. 4b) is shown in black lines. The protomer $\mathrm{B}$ active site binding pockets ( $\mathrm{S} 1, \mathrm{~S} 2, \mathrm{~S} 4, \mathrm{~S} 2^{\prime}$, and $\mathrm{S} 3^{\prime}$ ) and bound $\mathrm{B}^{\prime}$ substrate residues (italics) are labeled in panel $\mathbf{a}$. b-h Superposed drugs are shown in colored $\mathrm{cpk}$ representation with published names provided for each. PDB accession codes: 11a-6LZE, 11b-6MOK, 13b-6Y2G, telaprevir -7C7P, x0072-5R7Y, x0434-5R83, and x1392-5RFT. Drawings for each inhibitor can be found in Supplementary Fig. 8.

phenyl biaryl groups is likely to occupy the $\mathrm{S}^{\prime}$ subsite, overlapping the position of SARS-CoV-1 Phe309 (P3') or the equivalent SARS-CoV-2 Val (P3'; Fig. 5d). We note the only substitution between SARS-CoV-1 and -2 Mpro in the $\mathrm{S}^{\prime}$ siteA46S-is in close proximity to the phenyl biaryl and the Ser46 hydroxyl could be a unique site to engage for further development. Thus, the structures here in complex with the Cterminal sequence provide a template for structure-based design of inhibitors targeting the Phe-S2 and S3' sites, which could not be rationally designed using Mpro structures with the Leu-S2 active site conformation.

To this end, we have analyzed the SARS-CoV-2 Mpro active site structures of the crystallographic fragment screening effort by Walsh and colleagues ${ }^{5}$ that identified 71 noncovalent and covalent binding small molecules. Fragments were screened by soaking crystals with the same form as the substrate-free wildtype structure here. In these crystals, the empty active site resembles the more open Phe-S2 like conformation seen in complex with the C-terminal autocleavage site. Functional groups from both noncovalent and covalent bound fragments were observed to occupy the S2 subsite. Flexibility in S2 to accommodate binding was observed for some fragments, but the vast majority stabilized the Phe-S2 like conformation, with an aromatic functionality repeatedly observed to form hydrophobic interactions with Met49, mimicking the Phe305 (P2) interaction observed here (Fig. 5g, h). The preference for the Phe-S2 binding fragments could suggest that, although inducing the Leu-S2 like conformation was possible within the crystal, the limited binding interface of the small fragments used coupled with the initial PheS2 like starting structure of the substrate-free crystals used for soaking could skew the resulting binding toward this active site conformation. Several S2 site binding fragments also bind the S3' site (Fig. 5g, h). Further to the discussion above, these fragments could represent promising starting points for development, especially by combining with those observed to bridge S2 with other subsites, for example, x0434 with overlapping S2 bound benzyl ring and a pyridine ring binding the S1 subsite (Fig. $5 \mathrm{~g}$ ).

The structures presented here also provide information on active site solvation, the consideration of which is valuable for

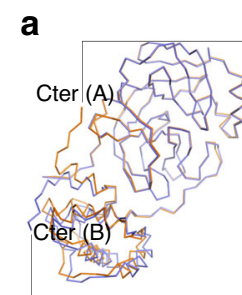

b
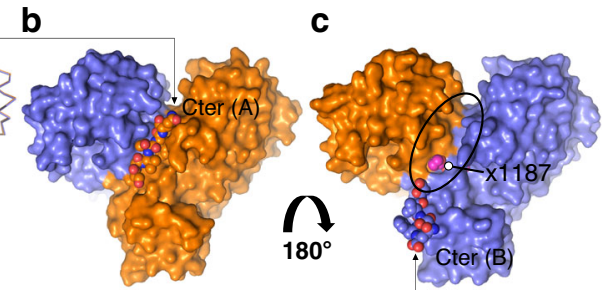

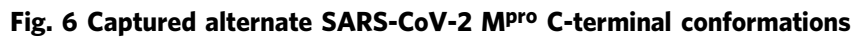
can inform drug discovery. a Superposition of SARS-CoV-2 Mpro acylenzyme intermediate protomers determined here with chain $A$ and chain $B$ in orange and blue, respectively. The alternate $C$-terminal orientationslabeled Cter (A) and (B)-observed reveal a druggable pocket at the dimerization interface. Arrows connect to corresponding $\mathrm{C}$-terminal orientation in (b) and (c). b The C-terminus of chain A (orange VdW representations) is packed at and stabilizes the dimerization interface (blue and oranges surfaces), an interaction typical of the mature dimer. $\mathbf{c}$ In the acyl-enzyme and product complexes, chain B redirects its C-terminus $\sim 180^{\circ}$ (blue $\mathrm{VdW}$ representations) as also shown in (a), allowing capture within the active site cleft of a neighboring dimer in the crystal, with the extended peptide binding groove at the dimerization site now exposed (delineated by black ellipse). A recent structure-based fragment screen found several small molecules bound within this region including compound $x 1187$ (magenta spheres; PDB 5RFA).

drug design. In addition to the well-ordered active site waters we observe, which are displaced by the C-terminal substrate (Fig. 2b), we also describe a putative deacylating water observed in both the acyl-enzyme intermediate and the empty active site protomer of the C145A mutant product complex (Fig. 2c). Interestingly, structures of alpha-ketoamide inhibitors, including 13b (ref. 7; Fig. 5e), and hepatitis $\mathrm{C}$ antivirals boceprevir (PDB 6WNP) and telaprevir (PDB 7C7P; Fig. 5f), show they position carbonyl oxygens superposing with both this catalytic water and the one occupying the oxyanion hole, allowing two direct hydrogen bond interactions with the catalytic center. Notable for boceprevir and telaprevir, the P1 cyclobutyl or propyl groups do not fill the $\mathrm{S} 1$ site, and two waters are present overlapping with the waters we observe displaced by the C-terminal Gln (P1) side chain amide deep in the S1 pocket (Fig. 5f). Extending the P1 moieties to displace these waters to bulk solvent could be a means to improve binding. In addition, and following on our prior discussion, we also note that for these promising alpha-ketoamides, which promote the Phe-S2 conformation of SARS-CoV-2, an extension of their $\mathrm{P}^{\prime}$ phenyl, amide, or cyclopropane groups into the adjacent $\mathrm{S}^{\prime}$ site would also be a possible design strategy for improved potency.

Finally, our structures also define a distinct binding site exposed due to the alternate positions of the C-terminal autocleavage sequence (Fig. 6a). In the mature enzyme here and in prior structures, Ser301-Gln306 typically pack at the dimerization interface, with Phe305 buried in a hydrophobic pocket defined by Phe8, Pro9, Ile52, Phe294, and the Arg298 propyl moiety of the same chain (Fig. 6b). This region is critical to dimerization and enzymatic activity, and many mutations affecting both map to this site (for review of these $s^{12}$ ), including mutation of Pro9 to threonine (P9T) identified and characterized here, which shows significantly diminished dimerization and activity (Supplementary Figs. 2-4). In the catalytic snapshots captured here, when the C-terminal autocleavage site is inserted into a neighboring dimer active site, this pocket becomes more solvent exposed (Fig. 6c) and is also modulated by the movement of domain III helix J (harboring Phe294 and Arg298; Supplementary Fig. 6a). Protein-protein interaction interfaces are being increasingly targeted for drug discovery ${ }^{34}$, and the essential 
role of oligomerization in $\mathrm{M}^{\text {pro }}$ activity suggests that targeting of the dimerization interface with small molecules that could inhibit self-association or interfere with the inter-subunit allosteric regulation of enzymatic activity represents a promising approach. In validation of this site as druggable, Walsh and colleagues identified two small molecules from a crystallographic fragment screen that were found to bind deep into the pocket ${ }^{5}$ (Fig. 6c).

In this context, the $\mathrm{M}^{\mathrm{p} r o}$ structures presented here with the $\mathrm{C}$ terminal autocleavage site bound as an acyl-enzyme intermediate or product form provides a C-terminal substrate-bound active site conformation that can be used to delineate atomic details of the mechanistic pathway, and optimize current inhibitor hits and design highly potent, novel Mpro inhibitors. We are currently exploring these drug design strategies with our recently described in silico deep docking methodology ${ }^{35}$.

\section{Methods}

Cloning, protein production, and purification. The gene encoding full-length SARS-CoV-2 Mpro with an additional N-terminal AVLQ and C-terminal GPHHHHHH was ordered from Twist Bioscience codon optimized for expression in Escherichia coli (Supplementary Table 3). The gene was cloned between the BamHI and XhoI restriction sites of plasmid pGEX-6P-1 (Supplementary Table 3) for expression of the protein with native $\mathrm{N}$ - and $\mathrm{C}$-termini ${ }^{11}$. Mutant $\mathrm{C} 145 \mathrm{~A}$ was generated using QuickChange site-directed mutagenesis (Supplementary Table 3). Mutant P9T was a point mutant from cloning the full-length gene. Sequences were confirmed by DNA sequencing.

Protein expression was carried out in E. coli BL21 (DE3). Cells were grown at $37^{\circ} \mathrm{C}$ in $\mathrm{LB}$ media supplemented with $0.1 \mathrm{mg} / \mathrm{mL}$ ampicillin. At $\mathrm{OD}_{600} \sim 1$, the temperature was reduced to $16^{\circ} \mathrm{C}$, and protein expression was induced with addition of IPTG to $1 \mathrm{mM}$. Cells were harvested after $5 \mathrm{~h}$, resuspended in lysis

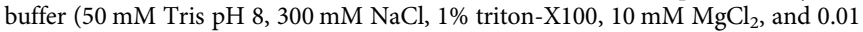
$\mathrm{mg} / \mathrm{mL}$ DNase I), and lysed with an Avestin Emulsiflex C5. The lysate was centrifuged at $50,000 \times g$ for $45 \mathrm{~min}$ and the soluble protein was loaded onto gravity flow column packed with $5 \mathrm{~mL}$ HisPur Ni-NTA resin (ThermoFisher Scientific) equilibrated in the lysis buffer with $20 \mathrm{mM}$ imidazole. The column was washed with ten column volumes of equilibration buffer, ten column volumes of the buffer with $40 \mathrm{mM}$ imidazole, and eluted with $50 \mathrm{mM}$ Tris $\mathrm{pH} 8,300 \mathrm{mM} \mathrm{NaCl}$, and 200 mM imidazole.

For the wild-type and P9T mutant protein, which cleave off the N-terminal GST tag during expression to leave the native $\mathrm{N}$-terminus, the eluate was concentrated by ultrafiltration (Amicon Ultra-30; Millipore Sigma) and the buffer was exchanged into $50 \mathrm{mM}$ Tris $\mathrm{pH} 8,300 \mathrm{mM} \mathrm{NaCl}$, and $1 \mathrm{mM}$ DTT to a final volume of $3 \mathrm{~mL}$. The C-terminal His-tag was removed with HRV 3C (Millipore Sigma) incubated overnight at $4{ }^{\circ} \mathrm{C}$. Uncleaved Mpro and the His-tagged HRV 3C were removed with a $0.5 \mathrm{~mL}$ HisPur Ni-NTA resin before further purification by gel filtration chromatography with a Sephacryl S-200 HR 16/60 column (GE Healthcare) equilibrated in $50 \mathrm{mM}$ Tris $\mathrm{pH} 7.4,1 \mathrm{mM}$ EDTA, and $1 \mathrm{mM}$ DTT.

Because the C145A mutant is inactive, wild-type, His-tagged Mpro was added to the eluate from the initial IMAC step at a 40:1 ratio, and the mixture was dialyzed overnight against $50 \mathrm{mM}$ Tris $\mathrm{pH} 8.0,300 \mathrm{mM} \mathrm{NaCl}$. The retentate was then incubated sequentially with $0.5 \mathrm{~mL}$ Glutathione Sepharose resin (GE Healthcare) equilibrated in dialysis buffer and $0.5 \mathrm{~mL}$ HisPur Ni-NTA agarose resin equilibrated in the same buffer with $20 \mathrm{mM}$ imidazole to remove the GST tag and His-tagged wild-type $\mathrm{M}^{\text {pro }}$, respectively, while the flow through and washes were collected. The C-terminal His-tag was removed with HRV 3C before further purification by gel filtration chromatography with a Superdex 200 Increase 10/300 GL column (GE Healthcare), as described above.

All proteins were concentrated to $10 \mathrm{mg} / \mathrm{mL}$ and frozen in liquid nitrogen for storage at $-80{ }^{\circ} \mathrm{C}$ until needed.

\footnotetext{
Analysis of protein quaternary structure. The molar masses of wild-type Mpro and the P9T variant were determined at $22^{\circ} \mathrm{C}$ with a size-exclusion chromatography system equipped with a Superdex 200 HR 10/300 GL column (GE Healthcare), an Agilent 1100 series HPLC pump and UV detector (Agilent Technologies), a Dawn Heleos II 16-angle light-scattering detection module and an Optilab T-rEX differential refractometer (Wyatt Technology). The mobile phase

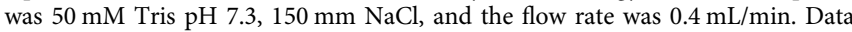
acquisition and analysis were achieved with Astra 6 software platform provided by Wyatt. The light-scattering detectors were normalized with monomeric bovine serum albumin (Sigma-Aldrich). A $100 \mu \mathrm{L}$ aliquot of each protein $(2 \mathrm{mg} / \mathrm{mL})$ was injected into the column at a time, and the column was washed with at least one column volume between samples. The protein absolute molecular mass was calculated assuming a specific refractive index increment $(\delta \eta / \delta c)$ value of $0.185 \mathrm{~mL} / \mathrm{g}$ and a theoretical extinction coefficient of $0.973 \mathrm{~mL} / \mathrm{mg} / \mathrm{cm}$.
}

BioSAXS data were collected with in-house X-rays (1.54 $\AA$ ) and a Rigaku bioSAXS-2000 (Rigaku Corporation). Scattering profiles of purified wild-type
SARS-CoV-2 Mpro were collected at 9.63, 4.82, 2.41, and $1.20 \mathrm{mg} / \mathrm{mL}$ and the P9T Mpro variant at $24.49,12.25,6.12,3.06,1.53$, and $0.77 \mathrm{mg} / \mathrm{mL}$. Twelve consecutive frames of $5 \mathrm{~min}$ in length were collected for each profile, corrected by subtracting the background scattering of the dialysis buffer ( $50 \mathrm{mM}$ Tris pH 7.4, $1 \mathrm{mM}$ DTT and $1 \mathrm{mM}$ EDTA), and normalized in concentration. Processing up to this point was carried out using SAXSLab (Rigaku Corporation). Further processing was performed with components of the ATSAS software package ${ }^{36}$. An extrapolated $0.00 \mathrm{mg} / \mathrm{mL}$ curve was generated for each of the samples. For the wild-type Mpro sample, the crystal structure of the Mpro dimer (PDB ID 6M03) was compared to the experimental data using CRYSOL ${ }^{37}$. For the P9T Mpro sample, chain A of the same structure was compared to the experimental data, with rather poor fit. As this result may stem from a slightly less restrained state of $M^{\text {pro }}$ as a monomer, SREFLEX $^{38}$ was used to allow for more flexible fitting of the PDB to the experimental data. To ensure that $\mathrm{NaCl}$ and DMSO were not affecting the dimerization state of Mpro, bioSAXS data for various samples of $1 \mathrm{mg} / \mathrm{mL}$ Mpro with the addition of $\mathrm{NaCl}$ and DMSO were collected, and processed as above. OLIGOMER $^{39}$ volume fraction analysis was used, with the $0.00 \mathrm{mg} / \mathrm{mL}$ extrapolated curves used as a basis for the dimer and monomer fractions. Full SAXS sample details, data collection parameters, software, structure parameters, and modeling statistics are listed in Supplementary Table 2.

Enzymatic activity. The protease activity of recombinant wild-type Mpro was assayed at $27^{\circ} \mathrm{C}$ with the FRET-based peptide substrate (MCA)AVLQ/SGFRLys (Dpn)-Lys- $\mathrm{NH}_{2}$ (GL Biochem, Shanghai) essentially as described $7,8,31,40$. The fluorescence of $7.5 \mu \mathrm{L}$ aliquots of this substrate in $50 \mathrm{mM}$ Tris buffer, $2 \mathrm{mM}$ EDTA $\mathrm{pH} 7.3$, and $10 \%$ DMSO was monitored with a BioTek Synergy H4 microplate reader $(330 \mathrm{~nm}$ excitation, $390 \mathrm{~nm}$ emission, and $9 \mathrm{~nm}$ slit band widths) for $3 \mathrm{~min}$ immediately before addition of $7.5 \mu \mathrm{L}$ of enzyme in the same buffer to start the reactions. For enzymological characterization the final Mpro concentration was $100 \mathrm{nM}$, while that of the substrate spanned the range between 0.5 and $100 \mu \mathrm{M}$. The initial rates of reaction, collected in triplicate at each substrate concentration, were determined from the linear regions observed during the first $3 \mathrm{~min}$ of each reaction. After correcting these values for the inner filter effect and converting to units of cleaved product as a function of time (i.e., $\mu \mathrm{M} / \mathrm{s}$ ) using a calibration curve constructed with (MCA)-AVLQ, these initial reaction rates were then subjected to nonlinear, least squares regression analysis with the Michaelis-Menten equation using the program OriginPro (OriginLab Corp., Northampton MA) to determine the kinetic parameters $K_{\mathrm{M}}$ and $k_{\text {cat }}$ and a Hill coefficient $n$, assuming a $100 \%$ active enzyme. Similarly, the dose-dependent inhibition of enzyme activity by Carmofur (Cayman Chemicals) was assayed to confirm that the recombinant Mpro behaves, as described in the literature. For this assay, the enzyme was incubated with different concentrations of Carmofur for $30 \mathrm{~min}$ before mixing with the substrate solution to monitor the residual activity also in at least triplicate. For this assay, the final enzyme and substrate concentrations were $30 \mathrm{nM}$ and $20 \mu \mathrm{M}$, respectively, while that of Carmofur spanned the range from $100 \mathrm{nM}$ to $30 \mu \mathrm{M}$.

Crystallization and structure determination. Crystals of the wild-type acyl enzyme or C145A mutant product complexes were obtained by sitting drop vapor diffusion using $0.8 \mu \mathrm{L}$ of protein $(\sim 10 \mathrm{mg} / \mathrm{mL})$ and $0.8 \mu \mathrm{L}$ reservoir containing 0.1 M MES pH 6, 10-16\% PEG 3350, and 5\% MPD. Crystals were cryoprotected by increasing the PEG 3350 concentration to $35 \%$ prior to freezing in liquid nitrogen. For the wild-type acyl-enzyme crystals, the cryoprotectant solution also included $2 \%$ DMSO. Wild-type substrate-free crystals were obtained as above from a reservoir containing $0.1 \mathrm{M}$ MES pH 6.5, 15-20\% PEG 3350, with streak seeding used to obtain diffraction quality crystals. Diffraction data were collected at $100 \mathrm{~K}$ on beamline 23-ID-B at the Advanced Photon Source (wild-type acyl-enzyme and substrate free; $1.03317 \AA$ wavelength), or on a Rigaku MicroMax 007 HF generator equipped with Osmic VariMax optics and a Dectris Pilatus3 R $200 \mathrm{~K}$ detector (C145A mutant; $1.5417 \AA$ wavelength). Both wild-type acyl-enzyme and C145A mutant product complex crystals belong to space group $C 2$ with isomorphous unit cell dimensions and two molecules in the asymmetric unit (Supplementary Table 1). The wild-type substrate-free crystals also belong to space group $C 2$ but with different crystal packing and only one monomer in the asym-

metric unit (Supplementary Table 1). The wild-type acyl-enzyme and substratefree data were processed using xia2 (ref. ${ }^{41}$ ) and $\mathrm{XDS}^{42}$, and the C145A mutant with the CrysAlis Pro software suite (Rigaku Inc.). Data reduction was carried out using Aimless ${ }^{43}$ as part of the CCP4 package ${ }^{15}$. The wild-type acyl-enzyme and product data exhibited anisotropy as assessed with the Diffraction Anisotropy Server ${ }^{44}$. Both non-truncated and truncated data were used in refinement and map calculations to assist interpretation. Phasing was carried using molecular replacement with Phaser ${ }^{45}$ as part of the CCP4 package, using PDB 6LU7 as a search model. Sequential rounds of model building and refinement were carried out using $\operatorname{Coot}^{46}$, Refmac ${ }^{47}$, and Buster ${ }^{48}$. Validation of the final models was carried out using MolProbity ${ }^{49}$ with excellent stereochemical model statistics, see Supplementary Table 1 . The wild-type acyl-enzyme intermediate model has a Molprobity score of 1.98 , clashscore of 4.16 and $96.88 \%$ Ramachandran favored, and $0 \%$ Ramachandran outliers. The C145A product complex has a Molprobity score of 1.74, clashscore of 2.67 and $97.04 \%$ Ramachandran favored, and $0.33 \%$ Ramachandran outliers. The wild-type substrate-free model has a Molprobity score of 
1.37, clashscore of 3.64 and $98.68 \%$ Ramachandran favored, and $0.33 \%$ Ramachandran outliers.

Secondary structure analysis was carried out using STRIDE ${ }^{50}$. Solvent accessible and buried surfaces were calculated using Areaimol ${ }^{15}$. All structure analysis and figure preparation was carried out with PyMOL (The PyMOL Molecular Graphics System, Version 2.1 Schrödinger, LLC) and Chimera ${ }^{51}$.

Docking. Before docking, protein structures were optimized using Protein Preparation Wizard module (Small-Molecule Drug Discovery Suite 2019-1, Schrödinger LLC, New York, NY, USA 2019). Docking grids were centered to the Cterminal substrates. Ligands were prepared using OpenEye's tautomers module, in order to assign the correct ionization and tautomeric form at $\mathrm{pH}$ 7.4 (QUACPAC 2.0.2.2. OpenEye Scientific Software, Santa Fe, NM, USA 2019). One low-energy 3D conformation was generated for each ligand, using Openeye's omega program in classic mode ${ }^{52}$. Docking was performed with Glide Single Precision module ${ }^{53}$.

Reporting summary. Further information on research design is available in the Nature Research Reporting Summary linked to this article.

\section{Data availability}

Structure factors and atomic coordinates have been deposited with the protein data bank with accession codes PDB ID 7KHP, 7JOY, and 7JP1. BioSAXS data have been deposited with SASBDB with accession code SASDJG5 and SASDJH5. Other data are available from the corresponding author upon reasonable request. Source data are provided with this paper.

Received: 8 August 2020; Accepted: 15 October 2020; Published online: 18 November 2020

\section{References}

1. Hilgenfeld, R. From SARS to MERS: crystallographic studies on coronaviral proteases enable antiviral drug design. FEBS J. 281, 4085-4096 (2014).

2. Yang, H. et al. The crystal structures of severe acute respiratory syndrome virus main protease and its complex with an inhibitor. Proc. Natl Acad. Sci. USA 100, 13190-13195 (2003).

3. Anand, K., Ziebuhr, J., Wadhwani, P., Mesters, J. R. \& Hilgenfeld, R. Coronavirus main proteinase (3CLpro) structure: basis for design of antiSARS drugs. Science 300, 1763-1767 (2003).

4. Fan, K. et al. Biosynthesis, purification, and substrate specificity of severe acute respiratory syndrome coronavirus 3C-like proteinase. J. Biol. Chem. 279, 1637-1642 (2004).

5. Douangamath, A. et al. Crystallographic and electrophilic fragment screening of the SARS-CoV-2 main protease. Nat. Commun. 11, 5047 (2020).

6. Kneller, D. W. et al. Structural plasticity of SARS-CoV-2 3CL M(pro) active site cavity revealed by room temperature X-ray crystallography. Nat. Commun. 11, 3202 (2020).

7. Zhang, L. et al. Crystal structure of SARS-CoV-2 main protease provides a basis for design of improved alpha-ketoamide inhibitors. Science 368, 409-412 (2020).

8. Jin, Z. et al. Structure of M(pro) from SARS-CoV-2 and discovery of its inhibitors. Nature 582, 289-293 (2020).

9. Jin, Z. et al. Structural basis for the inhibition of SARS-CoV-2 main protease by antineoplastic drug carmofur. Nat. Struct. Mol. Biol. 27, 529-532 (2020).

10. Ma, C. et al. Boceprevir, GC-376, and calpain inhibitors II, XII inhibit SARS$\mathrm{CoV}-2$ viral replication by targeting the viral main protease. Cell Res. 30, 678-692 (2020).

11. Xue, X. et al. Production of authentic SARS-CoV M(pro) with enhanced activity: application as a novel tag-cleavage endopeptidase for protein overproduction. J. Mol. Biol. 366, 965-975 (2007).

12. Goyal, B. \& Goyal, D. Targeting the dimerization of the main protease of coronaviruses: a potential broad-spectrum therapeutic strategy. ACS Comb. Sci. 22, 297-305 (2020).

13. Vuong, W. et al. Feline coronavirus drug inhibits the main protease of SARSCoV-2 and blocks virus replication. Nat. Commun. 11, 4282 (2020).

14. Paasche, A. et al. Evidence for substrate binding-induced zwitterion formation in the catalytic Cys-His dyad of the SARS-CoV main protease. Biochemistry 53, 5930-5946 (2014).

15. Winn, M. D. et al. Overview of the CCP4 suite and current developments. Acta Crystallogr. D Biol. Crystallogr. 67, 235-242 (2011).

16. Fodor, K. et al. Enzyme:substrate hydrogen bond shortening during the acylation phase of serine protease catalysis. Biochemistry 45, 2114-2121 (2006).

17. Burgi, H. B., Dunitz, J. D., Lehn, J. M. \& Wipff, G. Stereochemistry of reaction paths at carbonyl centres. Tetrahedron 30, 1563-1572 (1974).
18. Lee, J., Feldman, A. R., Delmas, B. \& Paetzel, M. Crystal structure of the VP4 protease from infectious pancreatic necrosis virus reveals the acyl-enzyme complex for an intermolecular self-cleavage reaction. J. Biol. Chem. 282, 24928-24937 (2007).

19. Chung, I. Y. \& Paetzel, M. Crystal structure of a viral protease intramolecular acyl-enzyme complex: insights into cis-cleavage at the VP4/VP3 junction of Tellina birnavirus. J. Biol. Chem. 286, 12475-12482 (2011).

20. Chung, I. Y. \& Paetzel, M. Crystal structures of yellowtail ascites virus VP4 protease: trapping an internal cleavage site trans acyl-enzyme complex in a native Ser/Lys dyad active site. J. Biol. Chem. 288, 13068-13081 (2013).

21. Alber, T., Petsko, G. A. \& Tsernoglou, D. Crystal structure of elastasesubstrate complex at -55 degrees C. Nature 263, 297-300 (1976).

22. Wilmouth, R. C. et al. Structure of a specific acyl-enzyme complex formed between beta-casomorphin-7 and porcine pancreatic elastase. Nat. Struct. Biol. 4, 456-462 (1997).

23. Katona, G. et al. X-ray structure of a serine protease acyl-enzyme complex at 0.95-A resolution. J. Biol. Chem. 277, 21962-21970 (2002).

24. Ding, X., Rasmussen, B. F., Petsko, G. A. \& Ringe, D. Direct crystallographic observation of an acyl-enzyme intermediate in the elastase-catalyzed hydrolysis of a peptidyl ester substrate: exploiting the "glass transition" in protein dynamics. Bioorg. Chem. 34, 410-423 (2006).

25. Vivares, D., Arnoux, P. \& Pignol, D. A papain-like enzyme at work: native and acyl-enzyme intermediate structures in phytochelatin synthesis. Proc. Natl Acad. Sci. USA 102, 18848-18853 (2005).

26. Tan, J. et al. pH-dependent conformational flexibility of the SARS-CoV main proteinase $(\mathrm{M}(\mathrm{pro}))$ dimer: molecular dynamics simulations and multiple $\mathrm{X}$ ray structure analyses. J. Mol. Biol. 354, 25-40 (2005).

27. Hsu, M. F. et al. Mechanism of the maturation process of SARS-CoV 3CL protease. J. Biol. Chem. 280, 31257-31266 (2005).

28. Xue, X. et al. Structures of two coronavirus main proteases: implications for substrate binding and antiviral drug design. J. Virol. 82, 2515-2527 (2008).

29. Muramatsu, T. et al. SARS-CoV 3CL protease cleaves its C-terminal autoprocessing site by novel subsite cooperativity. Proc. Natl Acad. Sci. USA 113, 12997-13002 (2016).

30. Świderek, K. \& Moliner, V. Revealing the molecular mechanisms of proteolysis of SARS-CoV-2 Mpro by QM/MM computational methods. Chem. Sci. 11, 10626-10630 (2020)

31. Dai, W. et al. Structure-based design of antiviral drug candidates targeting the SARS-CoV-2 main protease. Science 368, 1331-1335 (2020).

32. Jacobs, J. et al. Discovery, synthesis, and structure-based optimization of a series of N-(tert-butyl)-2-(N-arylamido)-2-(pyridin-3-yl) acetamides (ML188) as potent noncovalent small molecule inhibitors of the severe acute respiratory syndrome coronavirus (SARS-CoV) 3CL protease. J. Med Chem. 56, 534-546 (2013).

33. Turlington, M. et al. Discovery of N-(benzo[1,2,3]triazol-1-yl)-N-(benzyl) acetamido)phenyl) carboxamides as severe acute respiratory syndrome coronavirus (SARS-CoV) 3CLpro inhibitors: identification of ML300 and noncovalent nanomolar inhibitors with an induced-fit binding.

34. Mabonga, L. \& Kappo, A. P. Protein-protein interaction modulators: advances, successes and remaining challenges. Biophys. Rev. 11, 559-581 (2019).

35. Ton, A. T., Gentile, F., Hsing, M., Ban, F. \& Cherkasov, A. Rapid identification of potential inhibitors of SARS-CoV-2 main protease by deep docking of 1.3 billion compounds. Mol. Inform. 39, e2000028 (2020).

36. Petoukhov, M. V. et al. New developments in the ATSAS program package for small-angle scattering data analysis. J. Appl. Crystallogr. 45, 342-350 (2012).

37. Svergun, D., Barberato, C. \& Koch, M. H. J. CRYSOL- a program to evaluate $\mathrm{X}$-ray solution scattering of biological macromolecules from atomic coordinates. J. Appl. Crystallogr. 28, 768-773 (1995).

38. Petoukhov, M. V. \& Svergun, D. I. Global rigid body modeling of macromolecular complexes against small-angle scattering data. Biophys. J. 89, $1237-1250$ (2005).

39. Konarev, P. V., Volkov, V. V., Sokolova, A. V., Koch, M. H. J. \& Svergun, D. PRIMUS: a Windows PC-based system for small-angle scattering data analysis. J. Appl. Crystallogr. 36, 1277-1282 (2003).

40. Yang, H. et al. Design of wide-spectrum inhibitors targeting coronavirus main proteases. PLoS Biol. 3, e324 (2005).

41. Winter, G. xia2: an expert system for macromolecular crystallography data reduction. J. Appl. Crystallogr. 43, 186-190 (2010).

42. Kabsch, W. Xds. Acta Crystallogr. D Biol. Crystallogr. 66, 125-132 (2010).

43. Evans, P. R. \& Murshudov, G. N. How good are my data and what is the resolution? Acta Crystallogr. D Biol. Crystallogr. 69, 1204-1214 (2013).

44. Strong, M. et al. Toward the structural genomics of complexes: crystal structure of a PE/PPE protein complex from Mycobacterium tuberculosis. Proc. Natl Acad. Sci. USA 103, 8060-8065 (2006).

45. McCoy, A. J. et al. Phaser crystallographic software. J. Appl. Crystallogr. 40, 658-674 (2007). 
46. Emsley, P., Lohkamp, B., Scott, W. G. \& Cowtan, K. Features and development of Coot. Acta Crystallogr. D Biol. Crystallogr. 66, 486-501 (2010).

47. Murshudov, G. N., Vagin, A. A. \& Dodson, E. J. Refinement of macromolecular structures by the maximum-likelihood method. Acta Crystallogr. D Biol. Crystallogr. 53, 240-255 (1997).

48. Bricogne, G. et al. BUSTER version 2.10.3 (Global Phasing Ltd., Cambridge, United Kingdom, 2017).

49. Chen, V. B. et al. MolProbity: all-atom structure validation for macromolecular crystallography. Acta Crystallogr. D Biol. Crystallogr. 66, 12-21 (2010)

50. Heinig, M. \& Frishman, D. STRIDE: a web server for secondary structure assignment from known atomic coordinates of proteins. Nucleic Acids Res. 32, W500-W502 (2004).

51. Pettersen, E. F. et al. UCSF Chimera-a visualization system for exploratory research and analysis. J. Comput. Chem. 25, 1605-1612 (2004).

52. Hawkins, P. C., Skillman, A. G., Warren, G. L., Ellingson, B. A. \& Stahl, M. T. Conformer generation with OMEGA: algorithm and validation using high quality structures from the Protein Databank and Cambridge Structural Database. J. Chem. Inf. Model. 50, 572-584 (2010).

53. Friesner, R. A. et al. Glide: a new approach for rapid, accurate docking and scoring. 1. Method and assessment of docking accuracy. J. Med. Chem. 47, 1739-1749 (2004).

\section{Acknowledgements}

We thank GM/CA beamline staff at beamline 23-ID-B at the APS for access and support. GM/CA@APS has been funded in whole or in part with Federal funds from the National Cancer Institute (ACB-12002) and the National Institute of General Medical Sciences (AGM-12006). This research used resources of the Advanced Photon Source, a U.S. Department of Energy (DOE) Office of Science User Facility operated for the DOE Office of Science by Argonne National Laboratory under Contract No. DE-AC02-06CH11357. The Eiger $16 \mathrm{M}$ detector at GM/CA-XSD was funded by NIH grant S10 OD012289. We thank Martin Walsh, Diamond Light Source, for sharing of earlier reagents and protocols. This work was funded by operating grants from the Canadian Institutes of Health Research to N.C.J.S., M.P., and A.C. N.C.J.S. is a Tier I Canada Research Chair in Antibiotic Discovery.

\section{Author contributions}

M.V. performed all cloning experiments of WT, P9T, and C145A mutant Mpro. M.V., J.L., and F.I.R. performed protein purification. J.L. and L.J.W. performed crystallization, data collection, structure determination, and model building/refinement. M.P., L.J.W., J.L., and N.C.J.S. performed structural analysis. F.I.R. performed enzyme and inhibition assays, SEC-MALS analysis, and accompanying figures. N.A.C. performed BioSAXS experiments and accompanying figure. F.G., A.-T.T., F.B., and N.A.C. performed docking experiments. M.P. made all structure figures with input from L.J.W. and N.C.J.S. L.J.W., M.P., and N.C.J.S. wrote the manuscript with input from all authors.

\section{Competing interests}

The authors declare no competing interests.

\section{Additional information}

Supplementary information is available for this paper at https://doi.org/10.1038/s41467020-19662-4.

Correspondence and requests for materials should be addressed to M.P. or N.C.J.S.

Peer review information Nature Communications thanks the anonymous reviewer(s) for their contribution to the peer review of this work.

Reprints and permission information is available at http://www.nature.com/reprints

Publisher's note Springer Nature remains neutral with regard to jurisdictional claims in published maps and institutional affiliations.

(c) (i) Open Access This article is licensed under a Creative Commons Attribution 4.0 International License, which permits use, sharing, adaptation, distribution and reproduction in any medium or format, as long as you give appropriate credit to the original author(s) and the source, provide a link to the Creative Commons license, and indicate if changes were made. The images or other third party material in this article are included in the article's Creative Commons license, unless indicated otherwise in a credit line to the material. If material is not included in the article's Creative Commons license and your intended use is not permitted by statutory regulation or exceeds the permitted use, you will need to obtain permission directly from the copyright holder. To view a copy of this license, visit http://creativecommons.org/ licenses/by/4.0/.

(C) The Author(s) 2020 